\title{
How curriculum developers' cognitive theories influence curriculum development
}

\author{
Andrew Boudreaux (1)* \\ Department of Physics and Astronomy, Western Washington University, \\ Bellingham, Washington 98225, USA \\ Andy Elby 10 \\ Department of Teaching and Learning, Policy and Leadership and Department of Physics, \\ University of Maryland, College Park, Maryland 20742, USA
}

(Received 9 July 2019; accepted 23 December 2019; published 4 December 2020)

\begin{abstract}
[This paper is part of the Focused Collection on Curriculum Development: Theory into Design.] When we examined student responses to questions about the direction of the static friction force in various situations, we both had strong ideas about how to write a tutorial to promote deeper understanding. But our ideas were quite different. In this theoretical paper, we present the two contrasting tutorials and show how their differences can be traced to different theoretical orientations toward cognition and learning. We do not claim that one tutorial—or the theoretical framework loosely associated with it—is superior. Instead, we hope to illustrate two claims. One, we show in detail how curriculum designers' cognitive "theories" (frameworks), even if largely tacit during the act of creation, shape the resulting tutorials. Two, we show how, at least for us, articulating and discussing our respective theoretical orientations and their influence on our tutorial writing enables a rethinking of long-standing tutorial-writing habits. We argue that instructional intuition-shaped by explicit and tacit theoretical assumptions-functions well in guiding the design of curriculum, as our contrasting tutorials illustrate; but more systematic attention to the underlying theoretical assumptions can productively inform refinements.
\end{abstract}

DOI: 10.1103/PhysRevPhysEducRes.16.020144

\section{INTRODUCTION}

At a physics education research (PER) conference in 2005, Andrew B. was presenting a poster with data on students' reasoning about static friction. Andy E. came by, and the two of us got into a long discussion about how best to interpret the data. We roughly agreed about what pattern of thinking led many students to their incorrect answers. But Andy focused on what he thought was a productive intuition underlying those answers, while Andrew focused on the apparent failure of many students to systematically apply relevant physics principles. We started discussing how a tutorial might help students better understand static friction and quickly realized we would take appreciably different approaches, with the differences stemming at least in part from the different frameworks for cognition we were using to interpret the student data and structure our tutorials. We thought it would be interesting to write up

*andrew.boudreaux@wwu.edu

Published by the American Physical Society under the terms of the Creative Commons Attribution 4.0 International license. Further distribution of this work must maintain attribution to the author(s) and the published article's title, journal citation, and DOI. these ideas in a paper. Yes, that was 14 years ago. Luckily, this special issue kicked us into gear.

In this theoretical paper, we present the two contrasting tutorials and show how aspects of their differences can be traced to different assumptions about the nature of conceptual change and about the cognitive structures and mechanisms involved in the process. One tutorial foregrounds the desettling of incorrect conceptions and the building up of student understanding of the formal physics principles that support correct reasoning about the force of static friction. The other tutorial deemphasizes the physics first principles and instead guides students to refine their intuitions - the same intuitions that led to incorrect responses. We argue that the first tutorial corresponds roughly to a view of conceptual change in which problematic ideas, when challenged, can be destablilized, and students, when guided to revise (or even displace) those ideas, can adopt more normative physics principles. This view, influenced by but not equivalent to "classic" conceptual change theory [1], has more recently been articulated by Heron [2]. The second tutorial corresponds roughly to a knowledge-in-pieces cognitive framework [3], in which conceptual change corresponds to the (re)structuring of networks of primitive knowledge elements.

This is not a "paradigm war" article. We will not claim that one tutorial, or the cognitive framework loosely 
associated with it, is superior. Our point is to illustrate, in detail, how curriculum designers' theoretical orientations toward cognition and learning, even if largely tacit during the act of creation, can shape the resulting tutorials. We will then argue that post hoc conscious attention to the theoretical orientations underlying the tutorials can inform improvements. In our case, reflecting on, articulating, and then discussing our theoretical orientations as embedded in these static friction tutorials, including "trying on" one another's orientations, helped us come up with new ideas for tutorial revisions.

This is also not an empirical paper presenting a polished tutorial and evidence of its effectiveness. Instead, our goal is to increase the visibility of the messy, authentic process of curriculum development, by sharing details of our thinking about what we put into our tutorials and why, what we considered putting in but did not, and how we might modify the tutorials after the initial design. This thinking includes not only our interpretations of the empirical research and specific elements of our articulated theoretical orientations, but also our instructional intuitions-ideas about learning and teaching that are rooted in experience, and that often manifest during curriculum design as "what felt like the right way to go." We will argue that our theoretical orientations and instructional intuitions are not disjoint streams of knowledge that independently inform curriculum writing, but rather that they interact as they develop over time. Furthermore, both of our theoretical frameworks-and indeed, all learning theories-lack the detail and precision to fully determine what sequences of questions will maximize student learning. For these reasons, the influence of our theoretical frameworks on our tutorials is entangled with other influences in a complex way, and our tutorials are not simply "read out" from our frameworks. Still, we claim that the influence of our theoretical frameworks on our tutorials is identifiable and salient.

We hope to contribute to a long-standing though generally submerged discourse in the PER community about whether and how cognitive theory should inform curriculum development. In this ongoing discussion, some have foregrounded the generative role of theory [4], and others have emphasized the role of empirical data, without foregrounding explicit theoretical commitments [5]. We argue that instructional intuition along with thoughtful interpretation of student responses-shaped by explicit and tacit theoretical assumptions-function well in guiding the design of initial drafts of curriculum, as our contrasting tutorials illustrate, but that more systematic attention to and discussion of the underlying theoretical assumptions can productively inform refinements. While both of us teach in settings that lend themselves to small-group tutorials, we expect that the considerations put forth will have relevance to constructivist-informed curriculum development more broadly.

\section{BACKGROUND: RESEARCH ON STUDENT UNDERSTANDING OF STATIC FRICTION}

The research Andrew shared with Andy at the conference was conducted to explore student ideas about static friction forces in simple, real-world contexts. We summarize that research in this section, focusing on the data and brief interpretations shown on Andrew's posterthe artifact that launched us into our debates. Our purpose is to provide context for Secs. III and IV, which explore the "messy" process of curriculum development by describing how each of us interpreted the research through our contrasting theoretical lenses to arrive at two quite different tutorial drafts.

\section{A. Prior work of Close and Heron}

Andrew's research built on the examination by Close and Heron of student understanding of the static friction force in the introductory calculus-based mechanics course [6]. Close and Heron administered written questions in which a string exerts a horizontal force on a rectangular block that remains at rest on a horizontal surface. They found that some students related the static friction force directly to the surface area or the surface roughness. For example, some students answered that the static friction force would decrease if the block were reoriented from resting on its wide side to resting on its narrow side. Other students answered that the static friction force would increase when a piece of sandpaper is inserted between the block and the surface it is resting on. Many students seemed to equate the static friction force with $\mu N$, apparently not recognizing the possibility that $f<\mu N$.

\section{B. Context for instruction and research on student understanding}

Andrew's data came from a large enrollment, general education introductory physics course, intended for students not majoring in the sciences. There was no required textbook, and the mathematical demands were substantially lower than a typical calculus-based course. Course instruction emphasized that for an object to remain in the "at rest condition," the forces exerted on the object must balance. Static friction was introduced as a force exerted at and parallel to a surface of contact, with a magnitude that can take on whatever value is necessary to maintain force balance up to a certain upper limit, the "breaking point" of static friction.

\section{Research tasks and student responses}

Written questions inspired by the string and block tasks from Close and Heron were given on ungraded, in-class "pretests," graded in-class quizzes, and course homework. For brevity, we present only two of these questions, using them to illustrate some of the patterns in student reasoning that Andy and Andrew both found compelling at the 2005 
A magnet weighing $10 \mathrm{~N}$ is placed on the side of a refrigerator. A hand pushes upward with $6 \mathrm{~N}$ of force but the magnet does not move. Is the friction force exerted on the magnet upward, downward, or zero? Explain your reasoning.

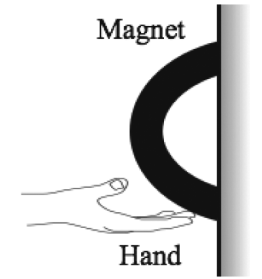

FIG. 1. The magnet question.

conference. For each question, we include a summary of the correct response and a discussion of the most common student responses. In Secs. III and IV, we each present our own interpretation of the data. We link these interpretations to specific theoretical orientations and discuss how the interpretations as well as our instructional intuitions guided decisions during curriculum development.

\section{Magnet question}

Description of question.-Figure 1 shows the "magnet question," given on an exam to 87 students. The students had received some instruction on friction interactions, including static friction, but had not completed any tutorial instruction on static friction. A variant of the magnet question was given to 67 students in another section of the course. The variant first asked students to draw a freebody diagram for the magnet, and only then asked explicitly about the direction of the friction force exerted on the magnet. The variant was administered as an ungraded, in-class written quiz.

To answer the magnet question correctly, a student can apply $\mathbf{F}_{\text {net }}=0$. The hand exerts an upward, $6 \mathrm{~N}$ force on the magnet, while Earth exerts a downward gravitational force of $10 \mathrm{~N}$. Together, these two forces create an unbalanced, downward force on the magnet of magnitude $4 N$. Thus, the static friction force exerted on the magnet by the refrigerator is upward, with magnitude $4 N$.

Results.-On the original version of the question (Fig. 1), one-quarter $(25 \%)$ of students gave a correct response. An additional $63 \%$ indicated that the friction force is directed downward, and $11 \%$ that the friction force is zero. On the variant, $16 \%$ answered correctly that the friction force is upward, $48 \%$ answered downward, and 34\% that the friction force is zero.

Discussion of common student reasoning.-(We note that the discussion here mirrors what was presented more briefly on Andrew's original poster.) The most common answer was that the friction force exerted on the magnet is vertically downward. One student explained as follows:

Friction always moves in the direction needed to prevent motion. Since the hand is trying to push the magnet upward, the friction force is exerted downward to prevent this motion.
In this response, the student does not explicitly refer to the second law or state that $\mathbf{F}_{\text {net }}=0$. No mention is made of a gravitational force exerted on the magnet-only friction and the hand are alluded to as force-exerting agents. The explanation is consistent with application of a simple "balancing" heuristic, in which the force applied by the hand is balanced by a friction force.

Of the students who gave this incorrect answer on the variant version of the question, $81 \%$ had included a downward gravitational or weight force on their free-body diagram. This suggests that most of the "downward friction" answers cannot be explained simply by a lack of awareness that the magnet experiences a downward gravitational force.

Another student's explanation for why the friction force points downward more strongly suggests the application of a "friction opposes agent" heuristic:

... if its force were up, with the hand, it would not be friction, it would help the push upward. We know it is not zero because... there must be a complementary force.

This response is consistent with identification of the hand as an active agent applying a push to the magnet, and with friction as a response or interaction that acts to oppose the hand's agency and keep the magnet from moving. This opposition to the hand seems central in the explanation, even forming the basis for classifying the force as a friction force.

Other student responses were similar, but less detailed:

\section{... friction forces act in directions opposite of the force imposed. \\ ... the law of inertia says that for objects at rest all opposing forces must be balanced.}

The opposing directions of a friction force and an "imposed" force is the focal aspect in these responses, as in the previous examples. Note that neither explanation states explicitly that all forces exerted on the magnet must sum to zero (i.e., the $\mathbf{F}_{\text {net }}=0$ condition).

A few responses offered additional insights into application of a "friction opposes external agent" heuristic. For example,

The friction force is exerted in both an up and down direction. I think this because there is clearly a friction force that is not allowing the hand to push the magnet up. This is a force downward. However, if the hand was not present, the magnet would still fall, so there is an upward friction force too.

This student explicitly identifies two separate friction forces. The downward friction force is linked, through an "opposition argument," to the upward force applied by the hand. A similar opposition argument, in this case involving 
Two teams play tug of war with a large box. Rope 1 has a tension of $110 \mathrm{lbs}$; rope 2, a tension of $95 \mathrm{lbs}$. The box

does not move.

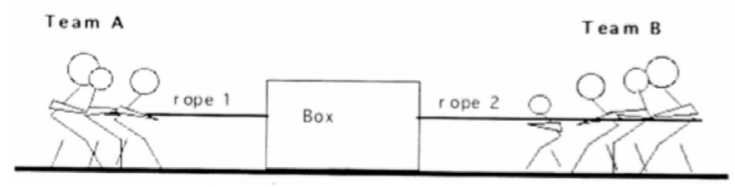

1. Draw a free-body diagram for the box.

2. Is the friction force exerted on the box to the left, to the right, in both directions, or zero? Explain your reasoning.

FIG. 2. The tug-of-war question.

the tendency of objects to fall, is implied when the student argues that "if the hand was not present, the magnet would still fall, so there is an upward friction force too." This type of response was rare, but interesting, suggesting that some students were viewing both the hand and gravity as external agents applying a force to the magnet, and were applying a heuristic that for an object to remain at rest, each applied force must be "balanced" or "canceled out" by a separate, opposing friction force. We wondered whether this type of reasoning might come up in other contexts as well.

\section{Tug-of-war question}

Description of question.-Figure 2 shows the "tugof-war question," given as an ungraded, written quiz to 68 students after instruction on forces and Newton's second law, but before any tutorial instruction on static friction.

To answer correctly, a student can recognize that for the box to remain at rest, the net force exerted on it must be zero. Since the tension force exerted to the left, by rope 1 , is stronger than the tension force to the right, by rope 2 , a static friction force must be exerted to the right.

Results.-Just under half the students (46\%) drew a correct free-body diagram for the box, showing two horizontal tension forces as a well as a single friction force to the right. Just over half (53\%) answered the second part correctly, stating that the friction force is to the right, and $38 \%$ of the students answered both parts correctly. The majority of students who answered correctly for the direction of the friction force in part 2 but did not draw a correct free-body diagram in part 1 simply omitted the friction force from an otherwise correct diagram. $18 \%$ of the students had a consistent response of $f=0$ on both parts of the question; these students did not draw a friction force on the free-body diagram, and also indicated explicitly on part 2 that the friction force is zero. Finally, $12 \%$ of the students had a consistent response of friction forces exerted in both horizontal directions on the two parts of the question, drawing two such force vectors on the free-body diagram in part 1 , and then stating explicitly in part 2 that there are friction forces in both horizontal directions. Table 1 summarizes these responses.
Discussion of common student reasoning.-On the tugof-war question, about $20 \%$ of the student responses indicated that two friction forces are exerted on the box, one in each horizontal direction. Many such responses included explanations similar to the following examples:

The friction force is going both ways. ... that is why the box is not moving. Each rope is fighting the friction and the tension of the other rope.

Friction is being exerted in both directions because the box is being pulled in both directions. If there were friction to one side or the other, then the box would be moving to one side or the other.

Each explanation links an individual friction force to a force applied by a single rope, through an oppositional relationship in which friction is "fighting" the rope. The explanations do not mention the $\mathbf{F}_{\text {net }}=0$ condition; instead, they account for the box remaining at rest through a one-to-one correspondence between an applied tension force and a responding friction force. These responses are

TABLE I. Student responses to the tug-of-war question $(N=68)$.

Part 2 (Is friction force on box to the left,

Part 1 (Free-body diagram) to the right, or zero?)

\begin{tabular}{|c|c|c|c|}
\hline Correct & $46 \%$ & Correct & $53 \%$ \\
\hline $\begin{array}{l}\text { No friction force (but drew } \\
\text { two tension forces, one } \\
\text { in each horizontal direction) }\end{array}$ & $38 \%$ & Zero & $18 \%$ \\
\hline $\begin{array}{l}\text { Friction forces in both } \\
\text { directions }\end{array}$ & $12 \%$ & $\begin{array}{l}\text { Both to the left } \\
\text { and to the right }\end{array}$ & $22 \%$ \\
\hline
\end{tabular}

Part 1 and part 2

Correct on both $38 \%$

Correct on part 2 but omitted $10 \%$ friction force on part 1

No friction force on part $1 \quad 18 \%$ and $f=0$ on part 2

Friction force in both directions on part 1 and on part 2 
similar to, but more common than, the pairwise balancing responses to the magnet question shared earlier.

An additional $\sim 20 \%$ of responses stated that the friction force is zero. The following explanations are representative:

[The friction force is zero]. ... because the box does not move. In order for there to be friction the box must be moving.

[The friction force is zero]. ... because the box is not moving, so it is not moving against anything causing friction.

\section{Discussion}

The magnet question and tug-of-war questions both involve an object in the "at rest condition." Physics experts recognize these tasks as similar, each involving equilibrium with all relevant forces specified except for static friction. These questions can all be explained by applying $\mathbf{F}_{\text {net }}=0$ to infer information about the static friction force. Indeed, these tasks were designed to explore whether students would apply this normative reasoning, and, if not, to gain insight into what approaches students do use. Well under half the responses showed clear evidence of the application of $\mathbf{F}_{\text {net }}=0$. Instead, many students seem to apply a heuristic that "friction opposes the external agent." Responses to the magnet question suggest that for some students, the hand has greater salience as an external agent than does Earth's gravity. For these reasons, we might expect the magnet question to pose a greater challenge. Indeed, the correct response rate was lower for the magnet question than for the tug-of-war question. Even on the tugof-war question, however, only about half of the responses gave the correct direction for the friction force.

\section{DESIGN OF TUTORIAL VERSION 1: AN APPROACH BASED ON THE DIFFICULTIES FRAMEWORK}

In this section, Andrew describes his interpretation of the research presented above, as well as his subsequent thinking about the design of a tutorial to support student learning. Explicit links are made to a particular cognitive framework-i.e., connections are drawn between curriculum development choices and a theoretical stance toward what is occurring cognitively as students learn and understand physics. In Sec. IV, Andy presents a parallel analysis of his curriculum development process.

\section{A. The difficulties framework}

At the time of the 2005 conference, I had been immersed for nearly a decade in use of a difficulties framework for making sense of student learning of physics. I had been exposed to this framework through my work as a graduate student with the Physics Education Group at the University of Washington (UW). As an undergraduate, in a traditional physics major curriculum, I had recognized, as many students surely do, that physics is a difficult subject. The UW group's approach to curriculum development, based on empirical investigations of how students apply specific concepts, excited me as a way to efficiently hone in on the locus of the learning challenge. The approach [7], which involves posing physics questions to students and attending carefully to their responses, was a way to find the "conceptual knots" [8] in the discipline, those areas that pose substantial difficulty for many learners. I thus view "difficulties" not as deficits or defects in the learner, but rather as areas of particular thorniness, subtlety, or complexity in the learning of the discipline itself. We know physics is hard; identifying specific difficulties serves to locate where the challenge resides.

Detailed knowledge of the conceptual knots gives rise to informed prescriptions for learning and teaching. Instruction can take students straight to the conceptual knots, challenging them to reflect on and modify their own thinking as they engage with normative physics reasoning through guided questions. The difficulties framework can be characterized as a "top down" approach, in that it juxtaposes common ideas and response patterns of novice learners with the "target" learning outcomes of a physics course. The approach emphasizes effortful learning, in which students are challenged to generate explanations that are both internally consistent and consistent with evidence and observations.

A common strategy taken in this guided approach to instruction, written about extensively elsewhere, is elicitconfront-resolve [9], a flexible algorithm for engaging students with a known conceptual knot. The elicit phase poses a question or asks students for a prediction to purposely draw out reasoning and ideas that differ from normative physics. This phase often makes use of tasks developed as part of the original research. Then, in the confront phase, students are pushed to develop conscious awareness of specific flaws in the argument they have just put forth. This can occur by drawing students' attention to two lines of reasoning, or to a line of reasoning and an observation, that should be consistent but are not. Finally, in the resolve phase, questions provide scaffolding for students to build a normative explanation, thus resolving the problematic aspects of the initial reasoning.

The difficulties framework, as a way of conceptualizing what happens during learning, involves a pair of fundamental assumptions. First, a degree of stability in student conceptions is assumed. The strategy of challenging and desettling non-normative ideas relies on those ideas maintaining coherence long enough to be held up, examined, and modified by the learner as they progress through the curriculum. In other words, for "confront" to be productive as an instructional move, there must be some cognitive entity for the learner to confront. Whether we refer to that entity as a heuristic, a mental model, an alternative conception, or something else, it must be long lived enough 
that learners themselves, as well as teachers, have something they can recognize and reflect on during instruction. Second, the approach assumes that learners generally prefer and seek out consistency as they make sense of new situations and ideas. For a learner to carry out the cognitive work of the resolve phase requires some level of drive to seek alignment between internal thinking and external evidence, a disposition referred to as epistemic rationality [10].

The difficulties framework can be regarded as a pragmatic orientation, employed in the service of increasing student facility with normative physics reasoning, rather than a mechanistic, detailed theoretical framework of the type that cognitive psychologists might develop and use to account for and predict specific learning phenomena. The difficulties framework is rooted in constructivist theories of learning. The seminal 2000 publication How People Learn connected constructivist theories to classroom practice in concrete ways [11]. The three key findings of How People Learn link the learner's prior knowledge and conceptual schemas, as well as the learner's metacognitive behaviors, to the quality of learning that can occur, providing clear underpinnings for the elicit-confront-resolve approach described above. In her 2003 Varenna lecture, Heron provides some details of these underpinnings and of how the difficulties framework can be used by a curriculum developer to link student responses on research tasks to specific instructional choices [12].

To summarize, a difficulties framework for student learning seeks to identify specific, fairly well-defined subdomains of normative physics that are challenging for many students to learn and understand. The framework assumes that students actively develop their own, at least semistable ideas when they engage with these subdomains, and that those ideas often differ in describable ways from the normative ideas. The framework assumes that during instruction, students tend to seek coherence in their thinking, by trying to align their ideas with external evidence. This view of student learning gives rise to the (somewhat) general instructional strategy of elicit-confront-resolve as a way to scaffold student learning in the context of a conceptual knot.

\section{B. How I interpreted the data}

The difficulties framework brings into relief specific contexts and lines of reasoning that are challenging-that is, specific conceptual knots in the discipline. Our research, and that of Close and Heron, had shown that one such conceptual knot involved making qualitative inferences about the direction or magnitude of the static friction force, based on knowledge of other forces acting on the object. A striking way in which the reasoning of novices seemed to diverge from normative reasoning was in application of the $\mathbf{F}_{\text {net }}=0$ condition to an object in equilibrium. Many students did not seem to be applying this fundamental principle, either as a first analysis or as a way to check an answer reached by some other means. Instead, many students seemed to employ an intuitively appealing heuristic, that a static friction force acts in opposition to a single applied force in order to keep an object at rest. This heuristic can yield answers consistent with Newton's second law in some cases, but can yield answers that conflict with Newton's second law for situations such as the magnet question, which involve two or more applied forces.

An additional finding stood out to me as an area of difficulty. On the sandpaper version of the string and block question, used in the Close and Heron study, some students had answered incorrectly that the friction force would increase when the sandpaper was added. I interpreted this response as potentially related to a failure to distinguish the static friction force from the maximum possible static friction force. My informal classroom experience, as well as the results from the magnet question and tug-of-war question, had suggested to me that part of the conceptual knot mentioned above involved the realization that the static friction force between two objects can change in magnitude in response to different applied forces. The sandpaper results seemed perhaps to indicate an extension or manifestation of that difficulty.

\section{Instructional strategy for the tutorial}

My primary goal for tutorial instruction was to engage students with the conceptual knot identified through research. I hoped to craft a sequence of questions that would guide students to apply $\mathbf{F}_{\text {net }}=0$ to an object at rest, in order to make inferences about the direction of the static friction force. I decided to start with a relatively simple physical context, and guide students in detail through an elicit-confront-resolve sequence, in hopes that they would recognize and adopt $\mathbf{F}_{\text {net }}=0$ as a "go to" strategy for objects in the at-rest condition. I would then ask students to apply the same reasoning, based on $\mathbf{F}_{\text {net }}=0$, to a more complicated situation, where I hoped that effortful cognitive work would solidify the learning. The research suggested two contexts, Close and Heron's block-andstring question and my tug-of-war question, that were well suited for this. (I decided that I would "save" the magnet question as a post-tutorial transfer task, to assess how effective the tutorial was in helping students instantiate the normative $\mathbf{F}_{\text {net }}=0$ reasoning.)

Figure 3 shows an extended excerpt from the tutorial. Section I of the tutorial is based closely on the work of Close and Heron, and I felt it would be ideal for my instructional goals [13]. The tutorial starts with the blockand-string context, asking students if, and how, the friction force changes when the block is reoriented (see part I.A in Fig. 3). I knew some students would answer "decrease"-a response that conflicts with $\mathbf{F}_{\text {net }}=0$. This would serve as the elicit phase-leading students right up to the conceptual knot. I also felt confident that the $\mathbf{F}_{\text {net }}=0$ idea, when 


\section{Static friction force}

A. A student conducts experiments with a block connected to a bucket of rocks with a cord and pulley. Experiments 1 and 2, at right, differ only in the orientation of the block. The system is at rest in each case.

Is the magnitude of the friction force exerted on the block in Experiment 2 greater than, less than, or equal to that in Experiment 1? Explain.

Friction forces can be categorized into two types: static friction and kinetic (or sliding) friction. A static friction force is one in which the two interacting objects are not moving relative to one another.

B. Sketch a free-body diagram for the block in Experiment 1 .

Rank the magnitude of the horizontal forces on your free-body diagram. Explain.

How, if at all, would the free-body diagram for the block be different in Experiment 2?

C. Revisit your answer to part A above. Is it consistent with your results from part B? If not, how could you change your answer to part A to make it consistent with part B?

\section{Breaking point}

A. Consider Experiment 1 again. You found that reorienting the block did not lead to a different amount of friction. Describe one way to change the situation that would result in a change in the friction force exerted on the block. Explain your reasoning.

B. Consider Experiment 1 again. Suppose you saw a sign that read "|f $f_{B T}^{(s)} \mid \leq 25 \mathrm{lbs} ;$ Add rocks at own risk." Explain the significance of the symbol " $\leq$ " in this situation.

Describe a method that you could use to check whether the sign is accurate or not. List the steps you would take and how you would interpret any measurements or observations in assessing your results.

In a given situation, the direction of the static friction force is opposite to the direction of the motion that would occur if friction were not present. In addition, there may be an upper limit to the magnitude that a static friction force can have. We will refer to the maximum possible magnitude of a static friction force as the breaking point.

C. Two teams play tug of war with a box that weighs $60 \mathrm{lb}$. Rope 1 has a tension of $120 \mathrm{lbs}$; rope 2, a tension of $95 \mathrm{lbs}$. The box does not move.

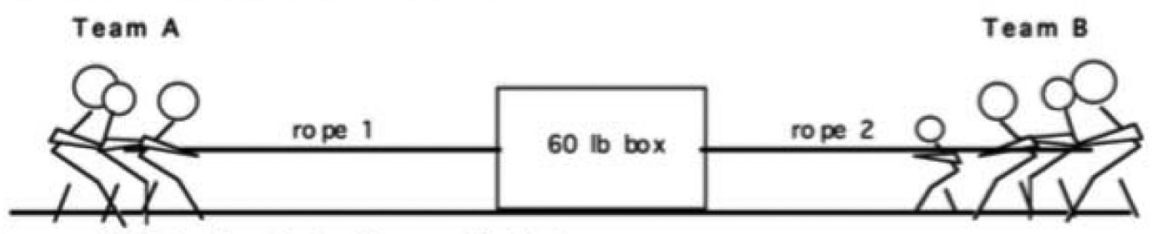

1. Draw and label a free-body diagram for the box.

2. What can be said about the breaking point between the box and the ground in this situation? Can any limits be placed on its value in this situation? Explain.

3. Suppose one of the members of Team A slips and falls, decreasing the tension in rope 1 by $35 \mathrm{lbs}$ (i.e from $120 \mathrm{lbs}$ to $85 \mathrm{lbs}$ ). Will the box start to move? Explain. How, if at all, would the free-body diagram for the box be modified?

FIG. 3. Extended excerpt from static friction tutorial based on the difficulties framework introduced, would be familiar and comfortable for many students — even if they had not on their own applied it to the block-and-string question. I assumed students would take this principle up as a learning resource, that, once activated, would provide a way for students to check the idea "less surface area means less friction" against another idea that they know to be valid. Thus, in part I.B, I ask students to sketch a free-body diagram for the block, to rank the 
magnitudes of the horizontal forces, and to compare the two cases of the block resting on its wider or its narrower side. These tasks pack in a lot of reasoning-I hoped that the social learning context would allow students more comfortable with the $\mathbf{F}_{\text {net }}=0$ principle to coach less comfortable students. This would serve as the confront phase-pushing students to become consciously aware of a contradiction between the "less surface area means less friction" idea and the $\mathbf{F}_{\text {net }}=0$ principle that had been previously emphasized in the course.

Part I.C of the tutorial serves as a way to resolve the contradiction. I hoped that the simple prompt to "check for consistency" would lead students who had initially thought the friction force decreases to recognize that this answer cannot be correct, because it conflicts with $\mathbf{F}_{\text {net }}=0$ for an object in equilibrium, a principle valid across all cases [14].

In Sec. II A of the tutorial, students are asked to think of a way to change the situation so that the static friction force would be different. This question in essence requires students to practice the $\mathbf{F}_{\text {net }}=0$ reasoning again, but now in reverse: instead of being given information about the applied forces, and asked about the effect on the friction force, students are given information about friction and asked to describe what change must have occurred in the applied forces. (During class, nearly all students here answered that changing the amount of rocks in the bucket should change the friction force.) I intended for this repetition with variation to strengthen students' uptake of $\mathbf{F}_{\text {net }}=0$ as a useful and general principle.

This question also sets up part II.B, in which students are prompted to develop the concept of $f_{\max }$. The research findings had suggested that students may not be fully distinguishing the static friction force from the maximum possible friction force. Other studies grounded in the difficulties framework have found that students struggle to distinguish closely related concepts [15]. By prompting them to explain the puzzling " $\leq$ " symbol in the equation for the static friction force, I expected that students would revisit and deepen their understanding of a central aspect of the static friction force, that it can vary in response to external circumstances. This, in turn, would provide a resource to help students resolve the identified difficulty of making an inference about the direction of the static friction force, elicited in the next section of the tutorial.

Part II.C of the tutorial provides an opportunity for students to apply the same $\mathbf{F}_{\text {net }}=0$ reasoning in a new, more complicated context: the tug-of-war scenario involves two applied forces from ropes, instead of the one such force in the block-and-string scenario. When asked to draw the free-body diagram, in part II.C.1, I hoped that students would try to apply $\mathbf{F}_{\text {net }}=0$, as they had been guided to do in part I. In doing so, students would recognize that now, the static friction force has a magnitude given by the difference in the magnitudes of the oppositely directed tension forces. In the process, I hoped students would reflect "Oh, $\mathbf{F}_{\text {net }}=0$ works here too," strengthening still further their understanding of Newton's second law as a universal principle. This application question does not scaffold the elicit-confront-resolve process with the same level of explicit detail as did Sec. I of the tutorial.

The research findings had shown that in the tug-of-war context, some students draw two friction forces, one opposing each of the two tension forces. As the prevalence of this response was about $20 \%$, I assumed that if the issue arose during tutorial, there would be some students in each collaborative group who would argue for the normative response, involving a net friction force.

Parts C. 2 and C. 3 of this section of the tutorial provide additional application practice with two difficult ideas students developed earlier in the tutorial. In part C.2, students must recognize that the actual static friction force may be less than the breaking point. Part C. 3 requires this idea, as well as application of the idea that static friction varies in response to external conditions.

\section{Summary: How the tutorial reflects the difficulties framework}

The difficulties framework leads curriculum developers to foreground student difficulties, i.e., gaps between normative reasoning approaches and common novice approaches. In the research described above, I tried to identify and describe in some detail productive reasoning that learners did not seem to be bring to bear. A primary gap was the failure to apply the $\mathbf{F}_{\text {net }}=0$ condition to an object that is remaining at rest and participating in a static friction interaction. Another gap was the failure to distinguish the magnitude of an actual static friction force from the maximum possible magnitude of such a force. In adopting the difficulties framework, I assumed that learners form or adopt cognitive structures that are at least semistable and that guide how they will respond to physics questions. In this case, students seemed to apply simple heuristics about how friction behaves, including more contact area means a greater friction force and for an object at rest, a static friction force balances an externally applied force. Finally, in conjunction with the difficulties framework, I focused my attention on the size and nature of the gaps between student reasoning approaches and normative approaches, more than on the continuity between these approaches. In other words, I focused explicitly, when developing the tutorial, on what students did not seem to be doing, more so than on the "seeds" of normative physics embedded in their reasoning.

Due at least in part to this stance toward cognition, I formulated a strategy for my tutorial: find specific contexts in which the heuristics give rise to different answers than the $\mathbf{F}_{\text {net }}=0$ approach, and focus student attention on these contexts in such a way as to draw out mistakes. The stringand-block scenario and tug-of-war scenario served as such contexts. I hoped the mistakes, when students were 
confronted with them, would then desettle the simple heuristics, providing an opportunity for learning. In my stance rooted in the difficulties framework, this learning would consist of the students replacing the heuristics with an application of $\mathbf{F}_{\text {net }}=0$. I felt this replacement would be possible because of another underlying theoretical assumption of the framework, that learners generally seek consistency between their thinking and external evidence. Prompts in the tutorial that ask students to compare their responses to two different questions (e.g., part I.C) reflect this assumption about the nature of learning.

\section{DESIGN OF TUTORIAL VERSION 2: AN APPROACH BASED ON THE RESOURCES FRAMEWORK}

\section{A. The resources framework}

At the time I saw Andrew's poster, I had been steeped in the resources (knowledge-in-pieces) framework [16] for about a decade, and it had influenced my instruction and curriculum writing, as I have argued elsewhere [17]. According to this framework, students' responses to physics questions do not always correspond to stable conceptions stored in students' heads. A classic illustration involves students' responses to "Why is it warmer in summer than winter?" Many students say it is because Earth is closer to the Sun in summer. From a resources perspective, however, we as researchers and curriculum developers should not assume that students "hold" this belief about Earth's orbit. Students may be constructing this "conception" on the spot, relying on cognitive resources developed through formal education, that Earth goes around the Sun, and on resources from lived experience relating to heat and light sources-perhaps as abstracted into the idea that closer is stronger [18]. These activated resources lead the student to conclude that Earth must be closer to the heat source (the Sun) in summer. By this account, the prevalence of this response does not reflect a stable conception about Earth's orbit in students' minds, but rather, reflects the salience of closer is stronger when students are considering a heat source and a question about why you feel hotter or colder.

From a resources perspective, learning is often a process of (re)organizing, (re)structuring, and refining knowledge elements you mostly or entirely already possess. For instance, in order to help students understand the seasons, a teacher might help students see continuity between closer is stronger and the idea that "head-on is stronger than glancing."

\section{B. How I interpreted the data}

At the 2005 conference, when I first encountered the magnet question and the resulting pattern of student responses, I thought, "oh, I bet the students are just thinking of friction as counteracting whatever other force they focus on," with contextual cues tipping students to focus on one force or another. So here, the diagram of the hand and/or the agentive nature of a pushing hand leads many students to focus on the upward force of the hand on the magnet as the force that friction must counteract. The examples of written responses were consistent with this interpretation: students wrote, "the friction force is exerted downward to prevent [the hand from pushing it upward]" and "if [the frictional] force were up, with the hand, it would not be friction, it would help the push upward. We know it is not zero because. ... there must be a complementary force." Even a low-prevalence answer was consistent with a "friction counteracts the force" interpretation: "The friction force is exerted in both an up and down direction. I think this because there is clearly a friction force that is not allowing the hand to push the magnet up. This is a force downward. However, if the hand was not present, the magnet would still fall, so there is an upward friction force too." By this account two separate friction forces are needed to counteract each of the two other influences exerted on the magnet.

Students' responses to the tug-of-war task also seemed, to me, to be consistent with my "friction counteracts the force" interpretation of how students think about the direction of a static friction force. In this scenario, neither tension force is more salient than the other. As compared to the magnet question, I expected fewer students to focus on just one or the other of the tension forces, and more students to focus on either the net force or on both forces separately (with a friction force counteracting each one). The response patterns matched these predictions: $45 \%$ instead of $30 \%$ gave the correct answer, and more than $15 \%$, instead of just $5 \%$, posited two frictional forces.

Note that friction counteracts the force does not explain why, on both tasks, many students wrote that friction is zero. My ad hoc explanation was that students "saw" opposing forces as already counteracting each other-they were attending to the direction but not the magnitude of the nonfrictional forces - and therefore saw no need for friction in a counteracting schema.

I want to emphasize that this interpretation of Andrew's data is not the result of a formal analysis using the knowledge-in-pieces framework. For instance, I am not trying to distinguish between abstract balancing, dynamic balancing, and canceling, three subtly different resources diSessa identifies [19]. To generate ideas for a tutorial, it is sufficient to recognize that many students are relying on some kind of "counteracting" schema, with friction counteracting some other force.

\section{Instructional strategy for the tutorial}

Unlike Andrew, I never created a tutorial for actual classroom use; at the time, I was not teaching undergraduate physics. But after my spirited discussion with Andrew and our formation of the idea for this paper, when I got back 


\section{Magnet on a refrigerator}

Consider the scenario shown - a magnet is stuck onto a refrigerator door. The magnet weighs $10 \mathrm{lbs}$, and a hand is pushing upward with a force of $6 \mathrm{lbs}$. The magnet remains at rest.

A. Relying on your gut intuition-your first thought about this scenario-decide whether the static frictional force exerted on the magnet (by the refrigerator) points upward, points downward, or equals zero. Explain your reasoning.

B. Now we'll guide you through some systematic reasoning.

1. Pretend, for the moment, that there's no friction in this scenario; the refrigerator door is perfectly slippery. Would the magnet move up, move

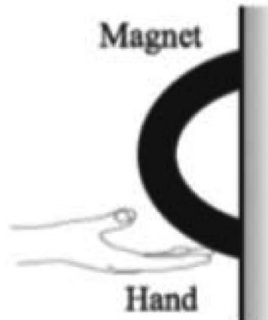
down, or stay in place?

2. So, in the actual scenario, what must be the direction of the friction force, to prevent the magnet from moving?

C. Compare your reasoning in part (A) with the reasoning of part (B). If they're not the same, how can your refine or modify your part (A) reasoning so that it agrees with part (B)?

\section{Tug-of-War}

Now consider a new scenario, pictured below. Two teams are playing tug-of-war with a large box. The tension in rope 1 is $120 \mathrm{lbs}$., the tension in rope 2 is $95 \mathrm{lbs}$., and the box weighs $60 \mathrm{lbs}$. The box is remaining at rest. For this scenario, you'll figure out not just the direction but also the magnitude of the friction force exerted on the object of interest, in this case the box.

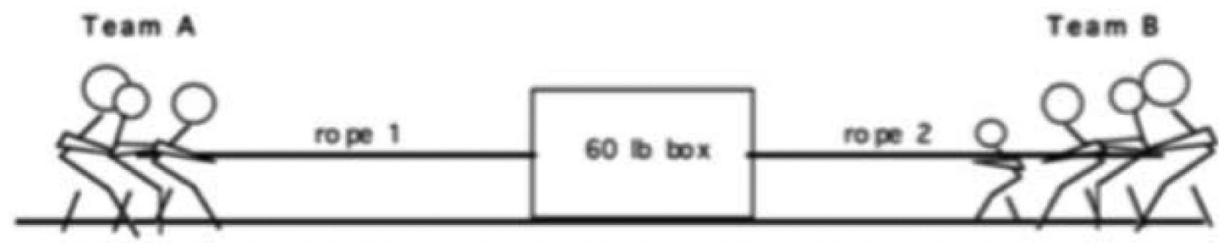

A. As quickly and intuitively as you can, guess the direction and strength (magnitude) of the force of static friction exerted on the box by the ground.

B. Let's again apply a particular systematic approach:

1. if the bottom of the box were perfectly slippery and therefore no frictional force were present, what would be the overall (net) force on the box - both its direction and its magnitude?

2. So, in the actual scenario, what must be the direction and magnitude of the friction force, to prevent the box from moving?

C. Compare your reasoning in part (A) with the reasoning of part (B). If they're not the same, how can your refine or modify your part (A) reasoning so that it agrees with part (B)?

III. Newton's $2^{\text {nd }}$ law

So far in this tutorial, we've been addressing forces and motion (or lack of motion, due to friction), but we haven't directly addressed the fundamental law relating forces and motion, Newton's $2^{\text {nd }}$ law $\left(\mathbf{a}=\mathbf{F}_{\text {net }} / \mathrm{m}\right)$.

A. Revisit the Magnet and Tug-Of-War scenarios from above. This time, explicitly use Newton's $2^{\text {nd }}$ law to solve for the magnitude and direction of friction force, in each of the two scenarios. Of course, your answers should agree with what you found above.

B. Here's a question more about problem-solving than friction. In I. and II. above, you used one or more semi-intuitive approaches to figure out the static friction force. Here, in III.A., you used a more formal approach based on Newton's $2^{\text {nd }}$ law. Looking back, would you say that the semi-intuitive approaches and the formal, Newtonian approach were different ways of getting to the same answer, or were they the same approach couched in different terms? There's no right answer to this - the point is just to consolidate the various ways of thinking about static friction you used in this tutorial.

FIG. 4. Draft of Andy's static friction tutorial based on the resources framework. 
to my room, I jotted down notes. Here is a cleaned-up version.

Given my previous work as a high school teacher [20] and later as a University of Maryland tutorial developer [21], I wanted to help students refine their everyday thinking, and to see themselves as doing so. Consistent with that bias, my strategy for the tutorial was to start with the friction counteracts force schema many students seemed to be activating, and help students develop their sense of which force friction fights against. Specifically, I wanted students to attend to the sum of the other (nonfrictional) forces - the "net other force"-and to conceptualize static friction as counteracting that force. Because I had the magnet and tug-of-war questions in my mind, I used those scenarios. Figure 4 shows a draft of the tutorial.

In part I.B. of the tutorial, the "trick"- which obviously must be tested to see if it works-is asking students what would happen in the absence of friction, as a way to make salient the net other force and the resulting motion. This trick emerges from instructional intuition; it is not "implied" by the resources framework. Still, the trick relies on a general implication of the resources framework, the idea that (re)focusing student attention on a particular aspect of the scenario can lead to different networks of activated resources, i.e., different ways of thinking about the scenario.

If the trick works-i.e., if students reason that with no friction present the magnet would slide down, and hence an upward static friction force is needed to counteract that tendency-then inviting students to continue using a counteracting schema, with friction counteracting the net other force, leads to correct reasoning and conclusions.

Part I.C. then invites students to reconcile their own gutfeeling way of thinking about static friction with the approach from part I.B, considering what would happen in the absence of friction.

Section II repeats that pedagogical flow, with the added complication of considering the magnitude, not just the direction of the forces. Finally, Sec. III invites students to think about how their earlier reasoning relates to a more formal approach starting with Newton's second law. My untested guess is that some students would see the intuitive approach as just an informal version of starting with Newton's second law, while others would see the two approaches as different but compatible-and this diversity in student views could spur a good epistemological discussion.

\section{Summary: How the tutorial reflects the resources framework}

The tutorial-writing process and product reflect the resources framework. Interpreting Andrew's data, I attended to and interpreted potentially productive cognitive resources-productive seeds-in students' incorrect reasoning, the friction counteracts force schema. Consistent with the resource framework, I viewed that cognitive resource as context dependent in whether it gets activated and what force gets mapped to the force in the schema. Then, writing the tutorial, I tried to help students refine that schema by "remapping" what force they plug into the schema; questions I.B.1 and II.B.1 try to help students see it as sensible, perhaps even intuitive, to schematize friction as counteracting the "net other force." Even when the tutorial finally nudges students to think about Newton's second law, it foregrounds the connection between that normative approach and the refined intuitive approach students develop (I hope) in Secs. I and II. In this way, my process and product reflect the resources-oriented approach to research as focusing on fine-grained, context-dependent resource activation and the resourcesoriented view of learning as (re)organizing, (re)structuring, and refining knowledge elements you mostly or entirely already possess.

\section{DISCUSSION}

\section{A. Summary of our main point}

As Secs. III and IV demonstrate, the differences between the two tutorial drafts arise, at least in part, from the different theoretical filters through which we interpreted students' reasoning about static friction on the magnet and tug-of-war questions, and different theoretically driven dispositions in our approaches to writing tutorials. We do not claim that we mechanically "applied" our theoretical frameworks to a given set of instructional goals and constraints. Instead, as we argue below, our dispositions and filters influence and are influenced by our student difficulties-based and resources-based theoretical frameworks. That is the primary mechanism by which our theoretical frameworks influence our tutorial writing.

Interestingly, to first order, both of us agreed about what reasoning led to the students' most common incorrect answers: students were viewing static friction as canceling or counteracting another salient force. But our different theoretical filters led us to different views of what cognitive structure was underlying that reasoning. Andrew viewed it as a comparatively robust, widely applied heuristic that does not reliably lead to correct responses. By contrast, Andy viewed it as the context-specific activation of a counteracting schema, a schema that students apply in various physics and nonphysics contexts, and which leads to correct or incorrect responses based on the context and on how various influences get "mapped" onto it.

These dispositions led us to different emphases in drafting our tutorials. Andrew focused on how students were not systematically using Newton's second law, and his difficulties-framework disposition was to help students apply physics principles to tackle a "conceptual knot." His tutorial guides students to take a systematic approach based on fundamental principles. Starting with Newton's 
second law, students recognize what the frictional force must be to ensure that $\mathbf{F}_{\text {net }}=0$. Andy focused on what he saw as a productive element of the student's reasoning, that friction must be counterbalancing something, and his resourcesframework disposition was to help students refine their intuitive, everyday thinking. His tutorial therefore scaffolds students in thinking more systematically-though not explicitly in terms of Newton's second law, until Sec. IIIabout what "force" friction is actually counteracting.

Other aspects of the tutorials, besides their common use of the student notion of "friction fights the salient force" as a basis for guided-inquiry instruction, are also similar. Both tutorials elicit and confront students' initial ideas and then guide them toward a resolution. (For example, see questions A and B in Secs. I and II of Andy's tutorial.) Both tutorials build on students' everyday reasoning. (For example, see questions II.A. and II.B in Andrew's tutorial.) So, elicitconfront-resolve and intuition refinement are pedagogical strategies in both the resources-based and difficulties-based tutorials. The difference between the theoretical frameworks and the associated tutorials lies in which of these strategies is most central and indispensable. From talking, we found that some of Andy's tutorials involve little or no confrontation, and some of Andrew's tutorials involve little or no explicit intuition building. Furthermore, as illustrated in detail above in Sec. III, even when a difficulties-perspective tutorial invites some intuition building, the confrontation of the student difficulty remains central to the conceptual flow. Conversely, even when a resources-based tutorial involves confrontation, the refinement of intuition remains central to the conceptual flow. Although we lack a formal definition of "central to the conceptual flow," we intend the two tutorials to illustrate differences between resources- and difficultiescentric curriculum.

At this point, we clarify our argument by responding to questions we anticipate this paper might engender (some of which come from anonymous reviewers).

1. Are you saying the tutorial writer's theoretical framework determines the tutorial they write?

No. First, we think the tutorial writer's theoretical orientation influences but does not determine the tutorial. Other influences include the writer's idiosyncratic teaching experiences and their effect on the writer's instructional intuitions, matters of personal style and taste, habits of mind forged by previous tutorial-writing experiences, and so on. Second, as illustrated above, the theoretical orientations, with their associated filters for interpreting data and dispositions for structuring tutorials, massively underdetermine the resulting curriculum. Two tutorial writers with similar theoretical orientations, presented with the same data on student thinking, would almost certainly not draft the same tutorial. Our point is just that some of the differences between Andrew's and Andy's tutorials are connected to our different theoretical orientations.

2. Are you saying your theoretical framework directly influences how your tutorial comes out?
If forced to choose whether the influence of our theoretical frameworks on our tutorials is primarily direct or primarily indirect, we will choose "indirect." We argue that the causal connection between a tutorial writer's theoretical framework and their tutorial writing is complicated. A writer's memories and schematizations of their experiences as a teacher and learner, their instructional intuitions, curriculum-writing dispositions and habits of mind, theoretical filters for interpreting data, and explicit theoretical frameworks are part of a cognitive complex system. In the years (or decades) before sitting down to draft a given tutorial, all these cognitive elements mutually affect each other as they develop over time. As a result, these elements are deeply interconnected, or even entangled. While this is speculative, we find it implausible that theoretical frameworks, instructional intuitions, and curriculum-writing habits of mind would develop independently of one other.

To further explore the notion of "direct" versus "indirect" effects of theoretical framework on curriculum design, we flesh out the developmental story. Over time, a writer's theoretical framework influences the writer's instructional habits of mind, which in turn influence their tutorial writing. And the writer's theoretical framework affects how they filter, interpret, and schematize their experiences as learners and as teachers. In this way, a direct effect of the writer's schematized teaching and learning experiences and instructional habits of mind on their tutorial writing also reflects an indirect effect of theoretical framework. Complicating this model, however, is the likelihood that the writer's teaching and learning experiences and instructional habits of mind affect the writer's theoretical framework through feedback loops. Teasing apart the direct and indirect effects of theoretical framework on tutorial writing, even if feasible analytically, would not reflect the underlying psychological reality-the underlying complex-systems causal network - of what is going on in the tutorial writer's head when they sit down to write a tutorial. We thus refine the paper's central claim as follows.

(i) A curriculum writer's theoretical framework can have noticeable effects on the curriculum. We support this claim with the noticeable differences in Andrew's and Andy's tutorials, differences that align with their differences in theoretical frameworks.

(ii) Given the complex-systems interconnections of the relevant cognitive elements, we cannot characterize the causal network of influences on tutorial writing in terms of direct and indirect effects. Therefore, we make no claims about whether a tutorial writer's experiences as a teacher and learner have "more influence" or "less influence" than the writer's theoretical framework.

3. Are you saying that one theoretical orientation is better than another for writing tutorials? 
No. As noted in the Introduction, this is not a "paradigm wars" article. And even if it were, we honestly think good tutorial drafts have emerged out of both paradigms. This bring us to the following question.

4. Which tutorial is better?

Our superficial answer is we do not know. Andy's draft of a tutorial segment-it is not even a complete tutorialhas never been classroom tested and refined. Nor did we present assessment data for Andrew's tutorial. (The data in Sec. II were collected before students worked through Andrew's tutorial.) We thus lack the assessment data needed to evaluate instruction. Our goal in this paper, however, is to show how the first drafts of the two tutorials reflect differences in our theoretical frameworks. Both first drafts would require iterations of testing and refinement to reach their potential.

Our deeper answer to "which tutorial is better" is that the question is ill defined. Since the two tutorials have different learning objectives, the tutorials could be "better" or "worse" on different measures. The two tutorials share a central learning objective: the ability to figure out the direction and magnitude of a static friction force in a scenario in which multiple forces act on a stationary object, including a functional understanding of the "adjustability" of static friction. After both tutorials undergo the usual rounds of testing and refinement, it would be fair to compare them using "near transfer" pre-post items involving such scenarios. We suspect that refined versions of both tutorials would achieve similar pre-post gains. (Indeed, an actual learning-outcomes comparison of tutorials written from a student-difficulties versus a resources orientation showed similar results [22].)

But the tutorials have other, nonshared learning objectives. Andrew's tutorial, unlike Andy's, explicitly aims to help students master a general problem-solving approach for figuring out forces on a stationary object, an approach that can be extended to objects in motion. We might expect Andrew's tutorial to produce better pre-post gains on "far transfer" items where, say, tension rather than friction provides the adjustable force on a stationary object. By contrast, Andy's tutorial, unlike Andrew's, has the explicit epistemological goal of helping students conceptualize physics thinking as the refinement of everyday thinking. We might expect Andy's tutorial to produce better pre-post gains on the items from epistemology-expectations surveys (like Colorado Learning Attitudes about Science Survey [23] or Maryland Physics Expectations Survey [24]) that probe the extent to which students think physics is supposed to make sense or cohere with their everyday experiences.

5. Could not those differences in learning goals, rather than your different theoretical frameworks, explain the differences between the two tutorials?

Certainly, some of the differences in the tutorials stem from the different learning goals. The clearest example is
Andy's explicit epistemology question (see part III.B of Andy's tutorial), reflecting his epistemological learning goals. By contrast, since both tutorials target similar conceptual learning goals, their different approaches to initiating the process of conceptual change must stem from other influences. And we argued above that differences in theoretical frameworks are one such influence.

Once again, cognitive complexity complicates the story. Learning goals are likely entangled with theoretical frameworks. For instance, Andy's epistemological goal of wanting students to see learning physics as the refinement of everyday thinking connects to the resources-based framework, which models learning as the restructuring and refinement of cognitive resources.

\section{B. Articulating and comparing theoretical orientations: Value added}

Even if we have established our main point, that a tutorial writer's theoretical orientation influences the tutorials they write, readers may ask why it matters. In writing curriculum, authors likely rely primarily on instructional intuition and habits of mind. While explicit theoretical frameworks are causally interwoven with those intuitions and habits, as argued above, a tutorial writer may not explicitly "call up" a framework during the creative act of generating a tutorial draft. And yet the PER community has produced a wealth of high-quality curricula over the past few decades. Furthermore, no matter how strongly a tutorial writer's theoretical perspective influences the first draft, the tutorial will go through multiple rounds of refinement that involve testing whether particular learning goals are met. As long as the eventual finished product produces adequate learning as defined by the assessment instrument, why does it matter how the first draft was produced?

We embarked on this paper partly to explore this question. Our experience has been that the combination of carefully articulating the theoretical assumptions and associated instructional choices underlying our tutorials and then comparing and discussing those assumptions has given us both ideas about how we might consider revising our tutorials going forward. And more generally, these discussions have led us to be more "meta" about what theoretical filters and predispositions we have chosen (consciously or unconsciously) when interpreting student thinking and drafting a tutorial. This allows us to be more purposeful, rather than habit driven, when thinking about which theoretical orientation might best serve a particular tutorial-writing task for a particular group of students. Here, we provide separate reflections along these lines.

\section{Andy's reflection on the value of articulating and reflecting upon theoretical frameworks}

I now think, after discussions with Andrew about his tutorial and the theoretical orientation underlying it, that my tutorial enacts a blasé attitude about relating students' 
intuitive thinking to Newton's second law. Section III of my tutorial, almost as an afterthought, has students check their reasoning against the second law, in the service of helping students see the formal physics as a refinement of everyday thinking. What is missing, Andrew helped me realize, is attention to another epistemological agenda I care about, helping students understand the power of physics to explain a lot in terms of a little. In the tutorial segment above, and in my tutorial drafts more generally, I emphasize my "seeing physics as the refinement of everyday thinking" agenda too narrowly, to the exclusion of other goals. Reworking the tutorial segment presented above, I would add a Sec. IV that introduces scenarios with stationary objects that are acted upon by other "adjustable" forces, like tension and normal forces. For each scenario, I would ask students to come up with an intuitive (to them) way of solving for the adjustable force, and then check whether their approach and solution "agree" with Newton's second law. After a few examples along those lines, I would ask students to reflect on whether they were using the same or different strategies for the different problems, and if they can articulate-to themselves and to another student-an approach that makes intuitive sense to them and would work for all the problems in the tutorial. Finally, I would ask students to reflect on whether and how that "general" strategy relates to Newton's second law.

Of course, those tutorial revisions might or might not work; like any tutorial draft, they would need to be classroom tested and refined over multiple iterations. My point is just that reflecting on my own theoretical orientation in tutorial writing-in particular, my predisposition for trying to help students refine their intuitive thinking - as well as talking with Andrew about his theoretical orientation, helped me "try on" another orientation more centered around foregrounding canonical physics. This enabled me to formulate tutorial revisions that I likely would not have come up with otherwise.

\section{Andrew's reflection on the value of articulating and reflecting upon theoretical frameworks}

After explaining my tutorial to Andy, I see ways I would like to change it. My tutorial opened by asking students to explain the changing surface area version of Close and Heron's string-and-block question. While this is a perfectly good context for eliciting problematic ideas, I realize now that starting instead with the sandpaper version of the task could allow the tutorial to not only desettle student conceptions, by confronting students with a contradiction between their answer and the normative $\mathbf{F}_{\text {net }}=0$ reasoning, but also to have students refine their initial ideas, and see those ideas as productive instead of just incorrect.
As discussed in Sec. II of this article, on the sandpaper version of the string-and-block question, many students answer incorrectly that the static friction force exerted on the block would increase when the sandpaper is added. This idea, while incorrect, is certainly not unreasonable. The purpose of sandpaper, after all, is to increase friction. As mentioned, the tutorial also developed the concept of breaking point. With the sandpaper context, students could be guided to recognize that although $\mathbf{F}_{\text {net }}=0$ implies that the static friction does not change, the breaking point does in fact increase when the sandpaper is added. This refinement of ideas could bolster students' confidence in their own thinking as productive, while also helping them instantiate Newton's second law as a "go to principle" for analyzing any object in the at-rest condition. It would provide a more satisfying resolve phase of the elicitconfront-resolve instructional approach, in which students could recognize both how the "friction increases" answer to the sandpaper question is flawed, being in conflict with $\mathbf{F}_{\text {net }}=0$, an how it can be seen to make sense, if redirected from the actual static friction force to the maximum possible friction force.

Discussion with Andy led me to consider one other major change, to Sec. II C of the tutorial. As described earlier, I had anticipated that some students, on the tugof-war free-body diagram, might draw two friction forces, one opposing each of the two tension forces. My assumption for the tutorial was that students would sort out this issue in their collaborative discussion. Now, however, I can imagine including an additional question, to confront and resolve this problematic response more explicitly, if it were to be elicited on the free-body diagram. Such a question would ask students to imagine they were standing on a skateboard, with one hand outstretched to the side, holding onto a nearby railing to keep themselves in place. Two friends are pulling on them, one forward and one backward. I would ask students to describe the direction of the force the railing would be exerting on them for situations in which the forces exerted by the two friends compared in different ways. I assume that imagining the kinesthetic experience of grasping the railing would support an answer in which the railing exerted a single force in one, welldefined direction. This answer would then serve as a resource to help students resolve the difficulty associated with the static friction force exerted on the box in the tug-of-war context. Ultimately, the decision about whether to introduce this new question would be made on the basis of how effective the original tutorial is in helping students recognize that the static friction force on the box by the surface is indeed exerted in a single, well-defined direction.

I summarize these reflections by noting that my first proposed change to the tutorial, to replace the changing surface area context with the sandpaper context, stemmed 
from me "taking up" Andy's theoretical orientation of helping students reorganize resources and refine their everyday thinking. My second proposed change, to include a new sequence involving the skateboard context, in contrast, came about as a result of me articulating my own theoretical orientation to Andy during our discussion.

\section{Summary: The value of articulating and reflecting upon theoretical frameworks}

Both of us found that articulating our own theoretical stance, and then discussing that stance with each other in the context of comparing and refining tutorials, helped us see ways to improve our tutorials. We speculate that much of the "value added" of articulating, reflecting upon, and discussing theoretical orientations (including formal frameworks and informal dispositions) may come in revision phases of tutorial writing.

However, we also contend that such articulation and reflection could be helpful during initial drafting, specifically to discover when a tutorial-writing habit has drifted away from one's theoretical framework. As mentioned above, Andy discovered such drift in his own tutorial-writing habits through reflection and discussion with Andrew: Andy's habit of focusing closely on helping students see learning physics as the refinement of everyday thinking had crowded out an equally important agenda stemming from his resources-based framework, namely the importance of helping students activate the same schema across multiple superficially different contexts as part of the resources refinementand-reorganization process. In principle, Andy could have discovered that drift before drafting his tutorial by reflecting on his tutorial-writing habits and his theoretical framework.

\section{CONCLUSION}

We have presented two different tutorials, each designed to promote student learning of static friction, and each informed by the same set of student responses to assessment questions. One tutorial was written by Andrew B., who adopted a difficulties framework in drafting the tutorial. The other was written by Andy E., who adopted a resources framework. The tutorials have many similarities, as well as some striking differences.

This paper has two takeaways. First, we illustrated how these theoretical frameworks, as manifested in both the "filters" through which we interpreted the data on student thinking and the predispositions with which we approach structuring a tutorial, help to make sense of the differences in our tutorial drafts. Second, we argued for the benefits of reflecting on, articulating, and discussing our theoretical orientations and how they influence our tutorial writing. The articulation and discussion, including "trying on" each others' orientations, helped us both come up with tutorial revision ideas we might not have formulated otherwise. We conclude that active metalevel reflection and discussion is not only intellectually interesting but also productive on a practical level for expanding the "idea space" from which a curriculum developer works.

\section{ACKNOWLEDGMENTS}

Material contributed to this manuscript by the first author (A. B.) is based on work supported by the National Science Foundation under Grants No. DUE1431857, No. DUE-1431541, No. DUE-1431940, No. DUE-1432765, No. DUE-1432052, No. DUE1821400, No. DUE-1821390, No. DUE-1821561, No. DUE-1821123, and No. DUE-1821511.
[1] G. Posner, K. A. Strike, P. W. Hewson, and W. A. Gertzog, Accommodation of a scientific conception: Toward a theory of conceptual change, Sci. Educ. 66, 221 (1982).

[2] P. R. L. Heron, Empirical investigations of learning, and teaching, Part I: Examining, and interpreting student thinking, in Proceedings of the Enrico Fermi Summer School on Physics Education Research, Varenna, Italy, 2013, edited by E. F. Redish and M. Vincentini (Italian Physical Society, Varenna, Italy, 2003); Empirical investigations of learning, and teaching, Part II: Developing research-based instructional materials, in Proceedings of the Enrico Fermi Summer School on Physics Education Research, Varenna, Italy, 2013, edited by E. F. Redish and
M. Vincentini (Italian Physical Society, Varenna, Italy, 2003).

[3] A. diSessa, Toward an epistemology of physics, Cognit. Instr. 10, 105 (1993).

[4] E. F. Redish, Millikan Lecture 1998: Building a science of teaching physics, Am. J. Phys. 67, 562 (1998).

[5] L. C. McDermott, Oersted medal lecture 2001: Physics education research-The key to student learning, Am. J. Phys. 69, 1127 (2001).

[6] H. G. Close, Improving instruction in mechanics through identification and elicitation of pivotal cases in student reasoning, Ph.D. thesis, University of Washington, 2005.

[7] For a cogent description, see Ref. [5]. 
[8] I, Andrew, trace my own use of the term "conceptual knot" to Stamatis Vokos, whom I have heard use it in various presentations. I am unaware of any formal definition of "conceptual knot" as a construct (private communication).

[9] See Refs. [2,5]; See also K. Wosilait, P. R. L. Heron, P. S. Shaffer, and L. C. McDermott, Development and assessment of a research-based tutorial on light and shadow, Am. J. Phys. 66, 906 (1998); P. S. Shaffer and L. C. McDermott, Research as a guide for curriculum development: An example from introductory electricity. Part II: Design of instructional strategies, Am. J. Phys. 60, 1003 (1992).

[10] K. E. Stanovich, What Intelligence Tests Miss: The Psychology of Rational Thought (Yale University Press, New Haven, CT, 2009).

[11] How People Learn: Brain, Mind, Experience, and School, edited by J. D. Bransford, A. L. Brown, and R. R. Cocking (National Academy Press, Washington, DC, 1999).

[12] See Ref. [2].

[13] See Ref. [6].

[14] In hindsight, I now see part I.C as a very weak resolve. After discussions with Andy, I came to feel that students might well be left with no clear way to reconcile competing ideas of "less contact area means less friction" and "F $\mathbf{F}_{\text {net }}=0$." See Sec. V for my ideas about how the tutorial can be modified.

[15] See, for example, D. E. Trowbridge and L. C. McDermott, Student understanding of the concept of velocity in one dimension, Am. J. Phys. 48, 1020 (1980).
[16] See Ref. [17]; See also D. Hammer, Student resources for learning introductory physics, Am. J. Phys. 68, S52 (2000).

[17] See D. Hammer and A. Elby, Tapping epistemological resources for learning physics, J. Learn. Sci. 12, 53 (2009); See also A. Elby and S. Yerdelen-Damar, Rethinking the relationship between instructors and physics education researchers, Phys. Rev. Phys. Educ. Res. (to be published).

[18] See Ref. [3].

[19] See Ref. [3].

[20] A. Elby, Helping physics students learn how to learn, Am. J. Phys. 69, S54 (2001).

[21] R. E. Scherr and A. Elby, Enabling informed adaptation of reformed instructional materials, AIP Conf. Proc. 883, 46 (2007).

[22] T. I. Smith and M. C. Wittmann, Comparing three methods for teaching Newton's third law, Phys. Rev. ST Phys. Educ. Res. 3, 020105 (2007).

[23] W. K. Adams, K. K. Perkins, N. S. Podolefsky, M. Dubson, N. D. Finkelstein, and C. E. Wieman, New instrument for measuring student beliefs about physics and learning physics: The Colorado Learning Attitudes about Science Survey, Phys. Rev. ST Phys. Educ. Res. 2, 010101 (2006).

[24] E. F. Redish, J. M. Saul, and R. N. Steinberg, Student expectations in introductory physics, Am. J. Phys. 66, 212 (1998). 